\title{
Positioning in Cellular Networks
}

\author{
Mirjana Simić and Predrag Pejović \\ University of Belgrade \\ Serbia
}

\section{Introduction}

Cellular networks are primarily designed to provide communication to mobile users. Besides the main application, determining location of mobile users (stations) within the cellular networks like Global System for Mobile Communications (GSM) and Universal Mobile Telecommunications System (UMTS) became an interesting additional feature. To provide the location based services (LBS), radio communication parameters already available in the network are preferably used, while some methods require investment in additional hardware to improve precision of the positioning. Positioning methods applied in cellular networks are characterized by tradeoff between the positioning precision and the requirements for additional hardware.

The idea to determine user location in cellular networks originated in the USA to support 911 service for emergency calls. The Federal Communications Commission (FCC) in 1996 initiated a program in which mobile operators are required to provide automatic location determination with specified accuracy for the users that make emergency calls. The new service is named Enhanced 911 (E-911). Similar service was initiated in Europe somewhat later, and it is called E-112. Besides the security related applications, availability of the user location information in cellular networks opened significant commercial opportunities to mobile operators.

In this chapter, methods to determine the mobile station position according to the radio communication parameters are presented. Position related radio communication parameters and their modeling are discussed, and algorithms to process collected data in order to determine the mobile station position are presented. Finally, standardized positioning methods are briefly reviewed.

\section{Position related parameters}

\subsection{Received signal strength}

According to wave propagation models (Rappaport, 2001), the received signal power may be related to distance $r$ between the mobile station and the corresponding base station by

$$
P(r)=P\left(r_{0}\right)\left(\frac{r_{0}}{r}\right)^{m}
$$

where $m$ is the path loss exponent, $r_{0}$ is the distance to a reference point, and $P\left(r_{0}\right)$ is the power at the reference distance, i.e. the reference power, obtained either by field measurements at $r_{0}$ 
or using the free space equation

$$
P\left(r_{0}\right)=\frac{\lambda^{2}}{(4 \pi)^{2} r_{0}^{2}} P_{t} G_{t} G_{r}
$$

where $\lambda$ is the wavelength, $P_{t}$ is the transmitted power, $G_{t}$ is the transmitter antenna gain, and $G_{r}$ is the receiver antenna gain. According to (2), the received signal power depends on the transmitter antenna gain, which is dependent on the mobile station relative angular position to the transmitter antenna. Also, (2) assumes direct wave propagation. In the case the wave propagation is direct, and the antenna gain is known, (1) may be used to determine the distance between the mobile station and the base station from

$$
r=r_{0}\left(\frac{P\left(r_{0}\right)}{P(r)}\right)^{\frac{1}{m}}
$$

which constitutes deterministic model of the received signal strength as a position related parameter.

The information provided by (3) may be unreliable in the case the antennas have pronounced directional properties and/or the propagation is not line-of-sight. In that case, an assumption that the received signal power cannot be larger than in the case the antennas are oriented to achieve the maximal gain and the wave propagation is direct may be used. Received signal power under this assumption locates the mobile station within a circle centered at the base station, with the radius specified by (3). This results in a probability density function

$$
p_{P}(x, y)=\left\{\begin{array}{cl}
\frac{1}{\pi r^{2}} & \text { for }\left(x-x_{B S}\right)^{2}+\left(y-y_{B S}\right)^{2} \leq r^{2} \\
0 & \text { elsewhere }
\end{array}\right.
$$

where $x_{B S}$ and $y_{B S}$ are coordinates of the base station, and $r$ is given by (3). This constitutes probabilistic model of the received signal power as a position related parameter.

\subsection{Time of arrival}

Another parameter related to mobile station location is the time of arrival, i.e. the signal propagation time. This parameter might be extracted from some parameters already measured in cellular networks to support communication, like the timing advance (TA) parameter in GSM and the round trip time (RTT) parameter in UMTS. Advantage of the time of arrival parameter when used to determine the distance between the mobile station and the base station is that it is not dependent on the whether conditions, nor on the angular position of the mobile station within the radiation pattern of the base station antenna, neither the angular position of the mobile station to the incident electromagnetic wave. However, the parameter suffers from non-line-of-sight propagation, providing false information of the distance being larger than it actually is. In fact, the time of arrival provides information about the distance wave traveled, which corresponds to the distance between the mobile station and base station only in the case of the line-of-sight propagation.

To illustrate both deterministic and probabilistic modeling of the information provided by the time of arrival, let us consider TA parameter of GSM systems. Assuming direct wave propagation, information about the coordinates of the base station $\left(x_{B S}, y_{B S}\right)$ and the corresponding TA parameter value TA localize the mobile station MS, $\left(x_{M S}, y_{M S}\right)$, within an annulus centered at the base station specified by

$$
T A R_{q} \leq r \leq(T A+1) R_{q}
$$


where $r$ is the distance between the base station and the mobile station

$$
r=\sqrt{\left(x_{M S}-x_{B S}\right)^{2}+\left(y_{M S}-y_{B S}\right)^{2}}
$$

and $R_{q}=553.46 \mathrm{~m}$ is the TA parameter distance resolution quantum, frequently rounded to $550 \mathrm{~m}$. The annulus is for $T A=2$ shown in Fig. 1.

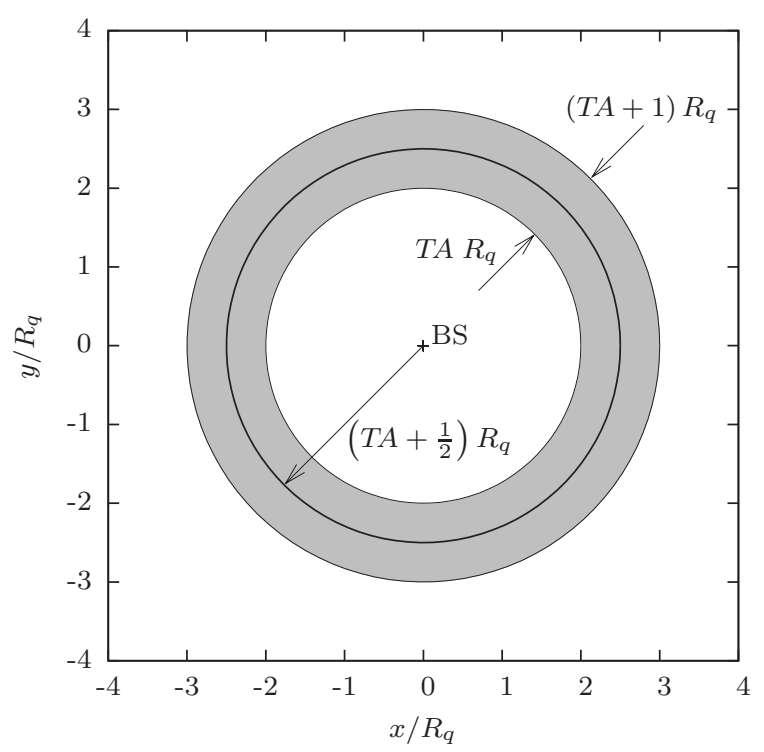

Fig. 1. Position related information derived from TA parameter, $T A=2$.

In a probabilistic model, the mobile station localization within the annulus is represented by the probability density function

$$
p_{T 1}(x, y)=\left\{\begin{array}{cl}
\frac{1}{\pi(2 T A+1) R_{q}^{2}} & \text { for } T A R_{q} \leq r \leq(T A+1) R_{q} \\
0 & \text { elsewhere. }
\end{array}\right.
$$

The probability density function of (7) assumes direct wave propagation, which is a reasonable assumption in some rural environments. However, in urban environments, as well as in some rural environments, indirect propagation of waves might be expected. As detailed in (Simić \& Pejović, 2009), in environments where indirect wave propagation might be expected the TA parameter value guarantees only that the mobile station is located within a circle

$$
r \leq(T A+1) R_{q}
$$

In absence of a better model, uniform distribution within the circle might be assumed, resulting in the probability density function

$$
p_{T 2}(x, y)=\left\{\begin{array}{cl}
\frac{1}{\pi\left((T A+1) R_{q}\right)^{2}} & \text { for } r \leq(T A+1) R_{q} \\
0 & \text { elsewhere. }
\end{array}\right.
$$


For both of the probability density functions, the area where the probability density functions might take nonzero value is limited to a square

$$
x_{B S}-(T A+1) R_{q} \leq x \leq x_{B S}+(T A+1) R_{q}
$$

and

$$
y_{B S}-(T A+1) R_{q} \leq y \leq y_{B S}+(T A+1) R_{q}
$$

which will be used to join the data collected from various information sources.

Choice of the probability density function that represents the information about the base station coordinates and the TA parameter value depends on the environment. The probability density function (7) should be used where the line-of-sight propagation is expected, while (9) should be used otherwise.

Deterministic model of the mobile station position information contained in the TA parameter value is much simpler. Assuming uniform distribution within the mobile station distance limits, and assuming the line-of-sight propagation, the distance between the mobile station and the base station is estimated as

$$
r=\left(T A+\frac{1}{2}\right) R_{q}
$$

The circle of possible mobile station location that results from $T A=2$ is shown in Fig. 1.

\subsection{Time difference of arrival}

To measure wave propagation time, clocks involved in the measurement should be synchronized. Term "synchronization" when used in this context means that information about a common reference point in time is available for all of the synchronized units. The requirement may be circumvented in time of arrival measurements if the round trip time is measured, which requires only one clock. Also, the requirement for mobile stations to be synchronized is avoided when the time difference of signal propagation from two base stations to the mobile station is measured. In this case, offset in the mobile station clock is canceled out, and only the base stations are required to be synchronized. The time difference of arrival might be extracted from time measurements on the Broadcast Control Channel (BCCH) or Traffic Channel (TCH) in GSM, or from SFN-SFN (System Frame Number) observed time difference measurements on the Common Pilot Channel (CPICH) in UMTS. Measured difference of the time of signal propagation results in information about the difference in distances between the mobile station and the two participating base stations. Value of the information provided by the time difference of arrival is not sensitive on the signal propagation loss, neither on the mobile station angular position, but suffers from non-line-of-sight wave propagation.

\subsection{Angle of arrival}

Historically, angle of arrival was the first parameter exploited to determine position of radio transmitters, as utilized in goniometric methods. The angle of signal arrival might be determined applying direction sensitive antenna systems. Application of specific antenna systems is the main drawback for application in cellular networks, since specific additional hardware is required. Besides, the information of the angle of arrival is not included in standardized measurement reports in cellular networks like GSM and UMTS. To extract useful information from the angle of arrival, line-of-sight propagation is required, again. Due to the drawbacks mentioned, positioning methods that utilize this parameter are not standardized for positioning applications in cellular networks yet. 


\section{Position estimation}

After the position related parameters are collected, position of the mobile station is determined by joining of the collected data applying some of the available methods. The methods can be classified as deterministic, probabilistic, and fingerprinting.

\subsection{Deterministic methods}

Deterministic methods apply geometric relations to determine position of the mobile station according to known coordinates of the base stations and distances and/or angles extracted from the radio parameters. The extracted distances and/or angles are treated as known, and uncertainty and/or inconsistency of the data are observed only when redundant measurements are available. In this section, geometric parameters extracted from the radio measurements and known coordinates of the base stations are related to the coordinates of the mobile station. It is assumed that the base stations, as well as the mobile station, are located in the same plane, i.e. that the problem is two-dimensional. All of the equations are derived for the two-dimensional case, and generalization to the three-dimensional case is outlined.

\subsubsection{Angulation}

To determine coordinates $\left(x_{M S}, y_{M S}\right)$ of a mobile station (MS) applying angulation method, at least two base stations are needed, BS1 and BS2, and their coordinates $\left(x_{B S k}, y_{B S k}\right), k \in\{1,2\}$ should be known. The only information base stations provide are the angles $\varphi_{k}, k \in\{1,2\}$ the rays (half-lines) that start from the base station BSk and point towards the mobile station form with the positive ray of the $x$-axis, $y=0, x>0$. The angles are essentially azimuth angles, except the azimuth angles are referred to the north, and the positive ray of the $x$-axis points to the east. The choice is made to comply with common notation of analytical geometry. The angle measurement is illustrated in Fig. 2, where the mobile station located at $\left(x_{M S}, y_{M S}\right)=$ $(5,5)$ is observed from three base stations, $\left(x_{B S 1}, y_{B S 1}\right)=(3,5)$ with $\varphi_{1}=0,\left(x_{B S 2}, y_{B S 2}\right)=$ $(5,2)$ with $\varphi_{2}=90^{\circ}$, and $\left(x_{B S 3}, y_{B S 3}\right)=(9,8)$ with $\varphi_{3}=-143.13^{\circ}$.

Coordinates of the base stations and the mobile station observation angles locate the mobile station on a line

which can be transformed to

$$
\frac{y_{M S}-y_{B S k}}{x_{M S}-x_{B S k}}=\tan \varphi_{k}
$$

$$
y_{M S}-x_{M S} \tan \varphi_{k}=y_{B S k}-x_{B S k} \tan \varphi_{k}
$$

if $\varphi_{k} \neq \pi / 2+n \pi, n \in Z$, i.e. $x_{B S k} \neq x_{M S}$. In the case $\varphi_{k}=\pi / 2+n \pi$ the equation degenerates to

$$
x_{M S}=x_{B S k} \text {. }
$$

The observation angle provides more information than contained in (13), locating the mobile station on the ray given by (14) and $x_{M S}>x_{B S_{k}}$ for $-\pi / 2<\varphi_{k}<\pi / 2$, or on the ray $x_{M S}<x_{B S_{k}}$ for $-\pi<\varphi_{k}<-\pi / 2$ or $\pi / 2<\varphi_{k}<\pi$. This might be used as a rough test of the solution consistency in the case of ill-conditioned equation systems.

To determine the mobile station coordinates, at least two base stations are needed. In general, two base stations $k \in\{1,2\}$ form the equation system

$$
\left[\begin{array}{ll}
\tan \varphi_{1}-1 \\
\tan \varphi_{2}-1
\end{array}\right]\left[\begin{array}{l}
x_{M S} \\
y_{M S}
\end{array}\right]=\left[\begin{array}{ll}
x_{B S 1} & \tan \varphi_{1}-y_{B S 1} \\
x_{B S 2} & \tan \varphi_{2}-y_{B S 2}
\end{array}\right]
$$




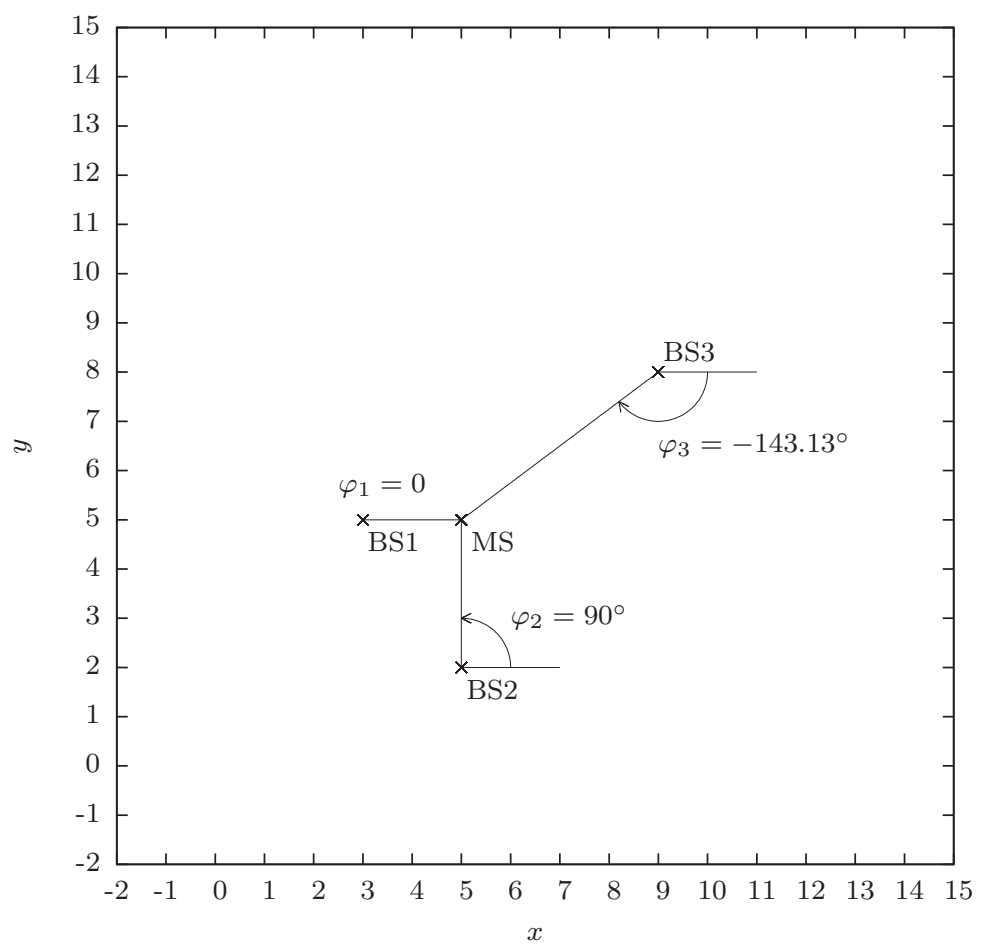

Fig. 2. Angulation.

assuming finite values for $\tan \varphi_{k}$. In the opposite case, corresponding equation should be replaced by an equation of the form (15).

In the case $\tan \varphi_{1}=\tan \varphi_{2}$, the base stations and the mobile station are located on the same line, and the equation system (16) is singular. An additional base station is needed to determine the mobile station coordinates, but it should not be located on the same line as the two base stations initially used. Furthermore, mobile station positions close to the line defined by the two base stations result in ill-conditioned equation system (16). This motivates introduction of additional base stations, and positions of three or more base stations on the same line, or close to a line, should be avoided.

In the example of Fig. 2, three base stations are available, and taking any two of the base stations to form (16) correct coordinates of the mobile station are obtained, since the systems are well-conditioned and the data are free from measurement error. If BS2 is involved, equation of the form (15) should be used.

In practice, more than two base stations might be available, and an overdetermined equation system might be formed,

$$
\left[\begin{array}{cc}
\tan \varphi_{1} & -1 \\
\vdots & \vdots \\
\tan \varphi_{n} & -1
\end{array}\right]\left[\begin{array}{l}
x_{M S} \\
y_{M S}
\end{array}\right]=\left[\begin{array}{c}
x_{B S 1} \tan \varphi_{1}-y_{B S 1} \\
\vdots \\
x_{B S n} \tan \varphi_{n}-y_{B S n}
\end{array}\right]
$$


where $n \in N$ and $n \geq 2$. The system (17) may be written in a matrix form

$$
\mathbf{A}\left[\begin{array}{l}
x_{M S} \\
y_{M S}
\end{array}\right]=\mathbf{b}
$$

The system (18) can be solved in a least-squares sense (Bronshtein et al., 2007), (Press et al., 1992) forming the square system

$$
\mathbf{A}^{T} \mathbf{A}\left[\begin{array}{l}
x_{M S} \\
y_{M S}
\end{array}\right]=\mathbf{A}^{T} \mathbf{b}
$$

To determine the mobile station location in three dimensions, coordinates of at least two base stations should be available in three-dimensional space, as well as two observation angles, the azimuth and the elevation angle. With the minimum of two base stations, two measured angles result in an overdetermined equation system over three mobile station coordinates. In practice, the two rays defined by their azimuth and elevation angles would hardly provide an intersection, due to the presence of measurement errors. Thus, linear least-squares solution (19) should be used even in the case only two base stations are considered. Let us underline that in three-dimensional case two base stations are still sufficient to determine the mobile station position.

A similar technique is applied in surveying, frequently referred to as "triangulation", since the object position is located in a triangle vertex, while the remaining two vertexes of the triangle are the base stations. Two angles are measured in order to determine the object position. The angles are frequently measured relative to the position of the other base station. Having the coordinates of the base stations known, the angles can be recalculated and expressed in the terms used here.

\subsubsection{Circular lateration}

Circular lateration is a method based on information about the distance $r_{k}$ of the mobile station (MS) from at least three base stations BSk, $k \in\{1, \ldots n\}, n \geq 3$. Coordinates $\left(x_{B S k}, y_{B S k}\right)$ of the base stations are known. An example for circular lateration using the same coordinates of the base stations and the mobile station as in the angulation example is presented in Fig. 3, where information about the mobile station position is contained in distances $r_{k}$ instead of the angles $\varphi_{k}$.

Let us consider a minimal system of equations for circular lateration

$$
\left(x_{M S}-x_{B S k}\right)^{2}+\left(y_{M S}-y_{B S k}\right)^{2}=r_{k}^{2}
$$

for $k \in\{1,2,3\}$. The equation system is nonlinear. According to the geometrical interpretation depicted in Fig. 3, each of the equations represents a circle, centered at the corresponding base station, hence the name of the method-circular lateration. If the system is consistent, each pair of the circles provides two intersection points, and location of the mobile station is determined using the information provided by the third base station, indicating which of the intersection points corresponds to the mobile station location. The problem becomes more complicated in the presence of measurement uncertainties, making exact intersection of three circles virtually impossible. An exception from this situation is the case where the two base stations and the mobile station are located on a line, resulting in tangent circles. In this case, the third base station won't be needed to determine the mobile station 


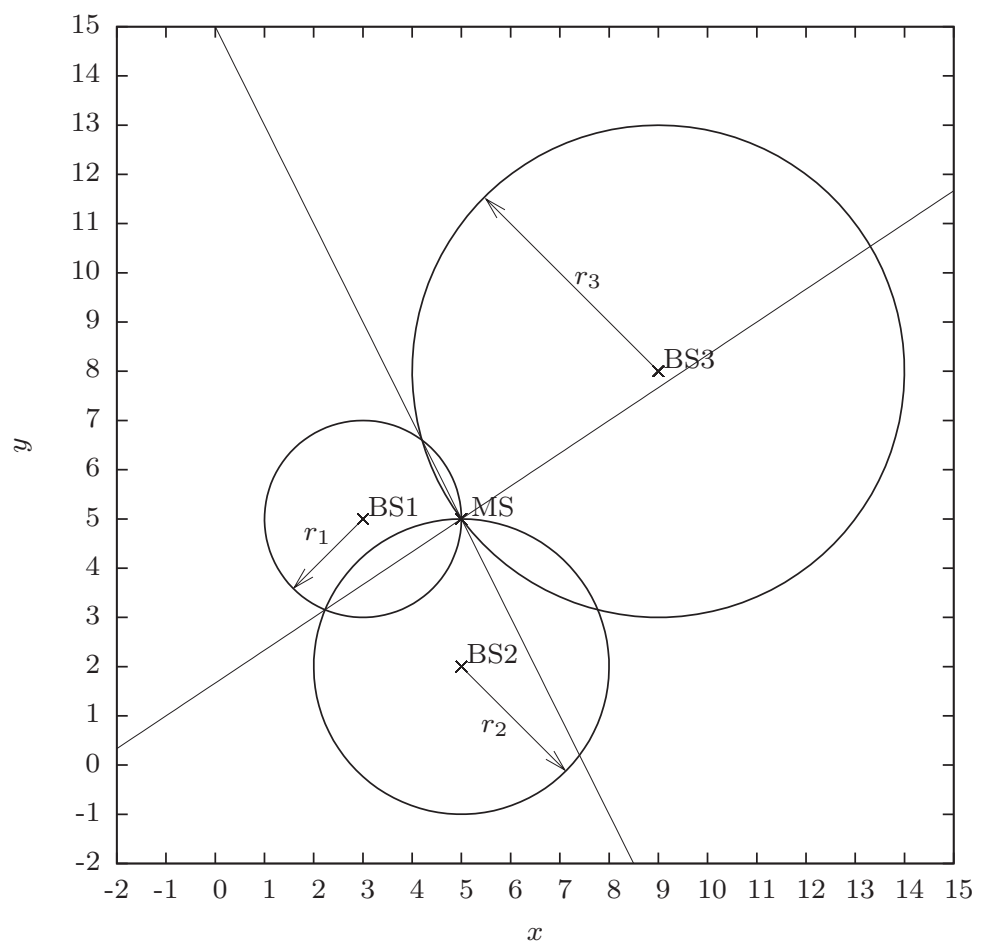

Fig. 3. Circular lateration.

position. Due to measurement uncertainty, it is also possible that measured distances result in circles that do not intersect.

The nonlinear system of equations (20) could be transformed to a linear system of equations (Bensky, 2008) applying algebraic transformations. This removes problems associated with solution methods for nonlinear equations and ambiguities about the mobile station location in the case the circle intersections that do not match. The first step in algebraic transformations is to expand the squared binomial terms

$$
x_{M S}^{2}-2 x_{M S} x_{B S k}+x_{B S k}^{2}+y_{M S}^{2}-2 y_{M S} y_{B S k}+y_{B S k}^{2}=r_{k}^{2} .
$$

Next, all squared terms are moved to the right-hand side

$$
-2 x_{M S} x_{B S k}-2 y_{M S} y_{B S k}=r_{k}^{2}-x_{B S k}^{2}-y_{B S k}^{2}-x_{M S}^{2}-y_{M S}^{2} .
$$

Up to this point, equations of the form (20) were subjected to transformation separately. Now, let us add the equation for $k=1$ multiplied by -1

$$
2 x_{M S} x_{B S 1}+2 y_{M S} y_{B S 1}=-r_{1}^{2}+x_{B S 1}^{2}+y_{B S 1}^{2}+x_{M S}^{2}+y_{M S}^{2}
$$

to the remaining two equations. Terms $x_{M S}^{2}$ and $y_{M S}^{2}$ on the right hand side are cancelled out, resulting in a linear system of two equations in the form

$$
2\left(x_{B S 1}-x_{B S k}\right) x_{M S}+2\left(y_{B S 1}-y_{B S k}\right) y_{M S}=r_{k}^{2}-r_{1}^{2}+x_{B S 1}^{2}-x_{B S k}^{2}+y_{B S 1}^{2}-y_{B S k}^{2}
$$


for $k \in\{2,3\}$. Each of the equations of the form (24) determines a line in the $(x, y)$ plane. The line equation is formed manipulating the equations of the circles centered at BS1 and BSk, and it is satisfied at both intersections of the circles, if the intersections exist. Thus, the line obtained from the two circle equations passes through the circle intersections. This geometrical interpretation is illustrated in Fig. 3 for both of the line equations (24).

The system of equations (24) could be expressed in a matrix form

$$
\left[\begin{array}{l}
x_{B S 1}-x_{B S 2} y_{B S 1}-y_{B S 2} \\
x_{B S 1}-x_{B S 3} y_{B S 1}-y_{B S 3}
\end{array}\right]\left[\begin{array}{l}
x_{M S} \\
y_{M S}
\end{array}\right]=\frac{1}{2}\left[\begin{array}{l}
r_{2}^{2}-r_{1}^{2}+x_{B S 1}^{2}-x_{B S 2}^{2}+y_{B S 1}^{2}-y_{B S 2}^{2} \\
r_{3}^{2}-r_{1}^{2}+x_{B S 1}^{2}-x_{B S 3}^{2}+y_{B S 1}^{2}-y_{B S 3}^{2}
\end{array}\right] .
$$

In the case all three of the base stations are located on the same line, their coordinates satisfy

$$
\frac{y_{B S 1}-y_{B S 2}}{x_{B S 1}-x_{B S 2}}=\frac{y_{B S 1}-y_{B S 3}}{x_{B S 1}-x_{B S 3}}
$$

which results in a singular system (25) since the determinant of the system matrix is zero. In that case, measurements from additional base stations should be used to determine the mobile station location, similar to the case the angulation method is applied and a singular system is reached. Also, base stations located in a close to a line arrangement result in ill-conditioned system (25) and huge sensitivity on the distance measurement error. Again, to avoid such situation additional base stations are needed, and their close to a line arrangement should be avoided as much as possible.

In the case measurements from $n$ base stations, $n \geq 3$, are available, the system of equations is overdetermined and it takes a matrix form

$$
\left[\begin{array}{cc}
x_{B S 1}-x_{B S 2} & y_{B S 1}-y_{B S 2} \\
\vdots & \vdots \\
x_{B S 1}-x_{B S n} & y_{B S 1}-y_{B S n}
\end{array}\right]\left[\begin{array}{c}
x_{M S} \\
y_{M S}
\end{array}\right]=\frac{1}{2}\left[\begin{array}{c}
r_{2}^{2}-r_{1}^{2}+x_{B S 1}^{2}-x_{B S 2}^{2}+y_{B S 1}^{2}-y_{B S 2}^{2} \\
\vdots \\
r_{n}^{2}-r_{1}^{2}+x_{B S 1}^{2}-x_{B S n}^{2}+y_{B S 1}^{2}-y_{B S n}^{2}
\end{array}\right] \text {. }
$$

This system takes the same compact form as (18), and should be solved as (19).

Presented analysis is performed under the assumption of consistent data, resulting in intersection of all circles in a single point. However, even in the case when only three base stations are considered (25) it is likely that the three circles do not intersect in the same point. The mobile station location is determined as an intersection of lines defined by intersections of pairs of circles. Obtained solution may be checked for compliance with the starting circle equations (20) to verify the solution and to estimate the error margins. This also applies in the case the linear least-squares method of (19) is applied to solve (27).

Presented method of linearization of the equations for the circular lateration method could be generalized to three-dimensional case in a straightforward manner. An additional base station would be needed to provide enough data to determine three unknown coordinates of the mobile station. The equation system would take the form similar to (25) and (27).

\subsubsection{Hyperbolic lateration}

Hyperbolic lateration is a method to determine the mobile station location applying information about differences in distance of the mobile station to a number of pairs of base stations with known coordinates. To determine the mobile station position this would require at least four base stations and information about at least three differences in distance.

To get acquainted with hyperbolic lateration, let us consider a case when the first base station (BS1) is located at $\left(x_{B S 1}, y_{B S 1}\right)=(c, 0)$, while the second base station (BS2) is located at 
$\left(x_{B S 2}, y_{B S 2}\right)=(-c, 0)$. It is assumed that $c>0$. Arbitrary locations of two base stations could be transformed to this case applying translation and rotation of the coordinates, as it will be discussed in detail later. The distance between the base stations is $D=2 c$. Let us also assume that a mobile station (MS) located at $\left(x_{M S}, y_{M S}\right)$ is for $d$ more distant from BS2 than from BS1, i.e.

$$
r_{2}-r_{1}=d
$$

where

$$
r_{1}=\sqrt{\left(x_{M S}-c\right)^{2}+y_{M S}^{2}}
$$

and

$$
r_{2}=\sqrt{\left(x_{M S}+c\right)^{2}+y_{M S}^{2}}
$$

are the distances between the mobile station and base stations BS1 and BS2, respectively. It should be noted that $d$ might either be positive or negative, being positive in cases when BS1 is closer to MS, and negative in the opposite case. According to the triangle inequality

$$
r_{2}+2 c>r_{1}
$$

and

$$
r_{1}+2 c>r_{2}
$$

which after algebraic manipulations limits $d$ to the interval

$$
-2 c<d<2 c \text {. }
$$

To provide convenient notation, let us introduce

$$
a=\frac{d}{2}
$$

which is according to (33) limited to

$$
-c<a<c .
$$

After the notation is introduced, the equation for the distance difference (28) becomes

$$
\sqrt{\left(x_{M S}+c\right)^{2}+y_{M S}^{2}}-\sqrt{\left(x_{M S}-c\right)^{2}+y_{M S}^{2}}=2 a .
$$

To remove the radicals, (36) has to be squared twice. After the squaring and after some algebraic manipulation, (36) reduces to

$$
\left(c^{2}-a^{2}\right) x_{M S}^{2}-a^{2} y_{M S}^{2}-a^{2}\left(c^{2}-a^{2}\right)=0 .
$$

At this point it is convenient to introduce parameter $b$ as

$$
b^{2}=c^{2}-a^{2}
$$

which reduces (37) to

$$
b^{2} x_{M S}^{2}-a^{2} y_{M S}^{2}-a^{2} b^{2}=0 .
$$

For $a \neq 0$ and $b \neq 0$, (39) might be expressed in a standard form

$$
\frac{x_{M S}^{2}}{a^{2}}-\frac{y_{M S}^{2}}{b^{2}}=1
$$


which is the equation that defines a pair of hyperbolas (Bronshtein et al., 2007).

Out of the two hyperbolas defined by (40), one is located in the right half-plane, $x>0$, which corresponds to the positive distance difference, $d>0$, while the one located in the left half-plane corresponds to $d<0$. In the case $d=0$, which implies $a=0$, according to (39) the hyperbolas degenerate to a line

$$
x_{M S}=0
$$

which specifies the set of points equally distant from BS1 and BS2.

Parametric description of a hyperbola that satisfies (39) is given by

$$
x_{M S}=a \cosh t
$$

and

$$
y_{M S}=b \sinh t
$$

where $t$ is a dummy variable. For $d>0$, i.e. $a>0$, the parametrically specified hyperbola is located in the right half-plane, while for $d<0$ it is located in the left half-plane, which meets the requirements of the physical model. Besides, the parametric description covers the degenerate case $d=0$, which is of interest in practice. In this manner, the set of points that are for $d$ more distant from BS2 than from BS1 is specified by

$$
x_{M S}=\frac{d}{2} \cosh t
$$

and

$$
y_{M S}=\frac{1}{2} \sqrt{D^{2}-d^{2}} \sinh t
$$

where $D$ is the distance between the base stations

$$
D=\sqrt{\left(x_{B S 1}-x_{B S 2}\right)^{2}+\left(y_{B S 1}-y_{B S 2}\right)^{2}} .
$$

This notation is convenient to plot the hyperbolas.

Far from the hyperbola center, which in the considered case is located at the origin $(0,0)$, in non-degenerate case $d \neq 0$ the hyperbola is approximated by its asymptotic rays

$$
y= \pm \frac{b}{a} x= \pm x \sqrt{\left(\frac{D}{d}\right)^{2}-1}
$$

which applies for $x>0$ if $d>0$ or for $x<0$ if $d<0$. Approximation of the hyperbola with these two asymptotic rays is helpful in estimating the number of intersections of two hyperbolas. Besides, it states that in the area far from the base stations, $r_{1}, r_{2} \gg D$, the information about the distance difference reduces to the angle of signal arrival, with some ambiguity regarding the asymptote that corresponds to the incoming signal, i.e. the sign of (47) which applies.

For application in hyperbolic lateration, arbitrary coordinates of the base stations must be allowed. This is achieved by rotation and translation of the hyperbola already analyzed. Let us assume that coordinates of the base stations are $\left(x_{B S 1}, y_{B S 1}\right)$ and $\left(x_{B S 2}, y_{B S 2}\right)$. The line segment that connects BS1 and BS2 is inclined to the ray $x=0, x>0$ for angle $\theta$ whose sine and cosine are

$$
\sin \theta=\frac{y_{B S 1}-y_{B S 2}}{D}
$$


and

$$
\cos \theta=\frac{x_{B S 1}-x_{B S 2}}{D} .
$$

Counterclockwise rotation for angle $\theta$ of the line segment that connects the base stations is achieved multiplying the vector of coordinates by the rotation matrix

$$
\left[\begin{array}{l}
x_{\text {new }} \\
y_{\text {new }}
\end{array}\right]=\left[\begin{array}{rr}
\cos \theta & -\sin \theta \\
\sin \theta & \cos \theta
\end{array}\right]\left[\begin{array}{l}
x_{\text {old }} \\
y_{\text {old }}
\end{array}\right] .
$$

Center of the hyperbola is located at the midpoint between the base stations

$$
\left[\begin{array}{l}
x_{C} \\
y_{C}
\end{array}\right]=\frac{1}{2}\left[\begin{array}{l}
x_{B S 1}+x_{B S 2} \\
y_{B S 1}+y_{B S 2}
\end{array}\right]
$$

After the rotation and the translation, the hyperbola is given by

$$
\left[\begin{array}{l}
x \\
y
\end{array}\right]=\left[\begin{array}{l}
x_{C} \\
y_{C}
\end{array}\right]+\left[\begin{array}{rr}
\cos \theta & -\sin \theta \\
\sin \theta & \cos \theta
\end{array}\right]\left[\begin{array}{lr}
a & \cosh t \\
b & \sinh t
\end{array}\right]
$$

which is after substitution expressed in positioning related terms as

$$
\left[\begin{array}{l}
x \\
y
\end{array}\right]=\frac{1}{2}\left[\begin{array}{l}
x_{B S 1}+x_{B S 2} \\
y_{B S 1}+y_{B S 2}
\end{array}\right]+\frac{1}{2}\left[\begin{array}{rr}
x_{B S 1}-x_{B S 2} & -y_{B S 1}+y_{B S 2} \\
y_{B S 1}-y_{B S 2} & x_{B S 1}-x_{B S 2}
\end{array}\right]\left[\begin{array}{c}
\frac{d}{D} \cosh t \\
\sqrt{1-\left(\frac{d}{D}\right)^{2}} \sinh t
\end{array}\right] .
$$

In some situations, it might be convenient to express the hyperbola by a single quadratic form over $x$ and $y$, instead of the two parametric equations. This can be achieved by substituting

$$
\cosh t=\frac{p^{2}+1}{2 p}
$$

and

$$
\sinh t=\frac{p^{2}-1}{2 p}
$$

and eliminating the dummy variable $p=e^{t}$ (thus, $p>0$ ) and one of the equations applying algebraic manipulations.

To illustrate hyperbolic lateration, the set of base stations BS1: $(3,5)$, BS2: $(5,2)$, BS3: $(9,8)$ and the mobile station positioned at $(5,5)$ are used, as shown in Fig. 4 . The same setup is already used to illustrate angulation and circular lateration. Differences in distances between the base stations and the mobile station are $d_{2}=d_{2,1}=r_{2}-r_{1}=1$ and $d_{3}=d_{3,1}=r_{3}-r_{1}=3$. These two differences in distance define two hyperbolas with focal points in BS1 and BS2, as well as BS1 and BS3, respectively, specified by

$$
-3 x^{2}+12 x y-8 y^{2}-18 x+8 y+25=0
$$

and

$$
-27 x^{2}-36 x y+558 x+216 y-2295=0 .
$$




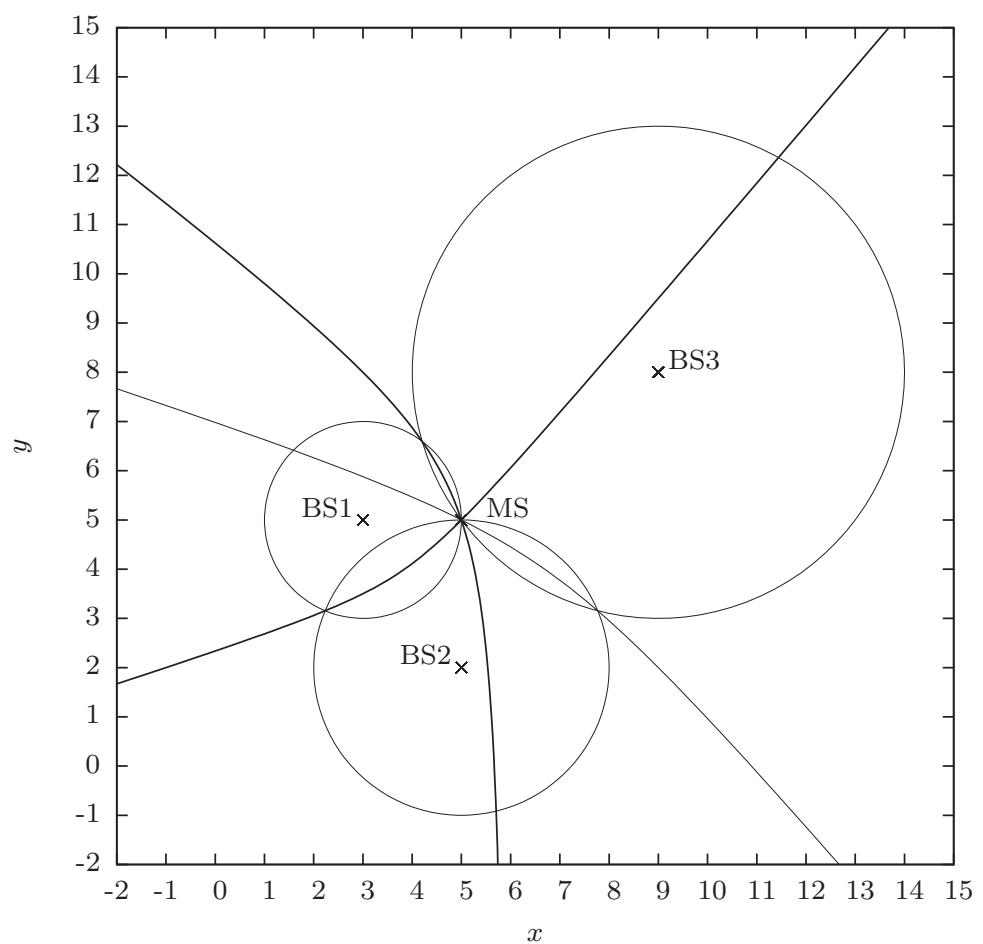

Fig. 4. Hyperbolic lateration, unique solution.

These hyperbolas pass through the intersections of circles defined by fixed distance between the base stations and the mobile station, similarly to the lines in the case of linearized system of equations for circular lateration (27). An additional difference in distance $d_{3,2}$ defined as

$$
d_{3,2}=r_{3}-r_{2}=\left(r_{3}-r_{1}\right)-\left(r_{2}-r_{1}\right)=d_{3}-d_{2}
$$

results in an another hyperbola

$$
-3 x^{2}-12 x y-8 y^{2}+102 x+164 y-755=0
$$

having focal points in BS3 and BS2, shown in Fig. 4 in thin line, but $d_{3,2}$ is linearly dependent on $d_{2}$ and $d_{3}$ and does not add any new information about the mobile station location.

It is possible to determine the mobile station location as an intersection of hyperbolas directly solving the nonlinear system of equations that arises from (28), like the system formed of (56) and (57). This approach requires iterative solution and raises convergence issues related to the numerical methods for nonlinear equation systems. However, it is possible to reduce the problem to a single quadratic equation or even to a system of linear equations applying appropriate algebraic transformations.

Let us start with the equation (24) derived for the method of circular lateration

$$
2\left(x_{B S 1}-x_{B S k}\right) x_{M S}+2\left(y_{B S 1}-y_{B S k}\right) y_{M S}=r_{k}^{2}-r_{1}^{2}+x_{B S 1}^{2}-x_{B S k}^{2}+y_{B S 1}^{2}-y_{B S k}^{2} .
$$


Instead of having distances $r_{k}$, distance differences

$$
d_{k}=d_{k, 1}=r_{k}-r_{1}
$$

are available. To eliminate the terms that involve $r_{k}$ consider

$$
r_{k}^{2}-r_{1}^{2}=\left(r_{1}+d_{k}\right)^{2}-r_{1}^{2}=2 d_{k} r_{1}+d_{k}^{2} .
$$

In this manner, $r_{k}$ is eliminated, while $r_{1}$ remains present in a linear term. After the transformation, the set of equations (60) becomes

$$
2\left(x_{B S 1}-x_{B S k}\right) x_{M S}+2\left(y_{B S 1}-y_{B S k}\right) y_{M S}=2 d_{k} r_{1}+d_{k}^{2}+x_{B S 1}^{2}-x_{B S k}^{2}+y_{B S 1}^{2}-y_{B S k}^{2}
$$

which for $k \in\{2,3\}$ results in the equation system expressed in a matrix form

$$
\mathbf{A}\left[\begin{array}{l}
x_{M S} \\
y_{M S}
\end{array}\right]=r_{1} \mathbf{b}_{1}+\mathbf{b}_{0}
$$

where

$$
\begin{gathered}
\mathbf{A}=\left[\begin{array}{ll}
x_{B S 1}-x_{B S 2} & y_{B S 1}-y_{B S 2} \\
x_{B S 1}-x_{B S 3} & y_{B S 1}-y_{B S 3}
\end{array}\right] \\
\mathbf{b}_{1}=\left[\begin{array}{l}
d_{2} \\
d_{3}
\end{array}\right]
\end{gathered}
$$

and

$$
\mathbf{b}_{0}=\frac{1}{2}\left[\begin{array}{l}
d_{2}^{2}+x_{B S 1}^{2}-x_{B S 2}^{2}+y_{B S 1}^{2}-y_{B S 2}^{2} \\
d_{3}^{2}+x_{B S 1}^{2}-x_{B S 3}^{2}+y_{B S 1}^{2}-y_{B S 3}^{2}
\end{array}\right] .
$$

Solution of the linear system is

$$
\left[\begin{array}{l}
x_{M S} \\
y_{M S}
\end{array}\right]=\mathbf{A}^{-1} \mathbf{b}_{1} r_{1}+\mathbf{A}^{-1} \mathbf{b}_{0}
$$

where $r_{1}$ is not known yet, while coordinates of the mobile station are provided as linear functions of $r_{1}$

$$
x_{M S}=k_{x} r_{1}+n_{x}
$$

and

$$
y_{M S}=k_{y} r_{1}+n_{y} .
$$

The value of $r_{1}$ is computed from

$$
\left(x_{M S}-x_{B S 1}\right)^{2}+\left(y_{M S}-y_{B S 1}\right)^{2}=r_{1}^{2}
$$

which after substitution of (69) and (70) results in a quadratic equation

$$
\left(k_{x}^{2}+k_{y}^{2}-1\right) r_{1}^{2}+2\left(k_{x}\left(n_{x}-x_{B S 1}\right)+k_{y}\left(n_{y}-y_{B S 1}\right)\right) r_{1}+\left(n_{x}-x_{B S 1}\right)^{2}+\left(n_{y}-y_{B S 1}\right)^{2}=0 .
$$

In general, the quadratic equation provides two solutions. If the equation corresponds to the physical model, at least one of the solutions should be positive. Possible negative solution for $r_{1}$ should be rejected for the lack of physical meaning. However, it is possible to obtain two positive solutions for $r_{1}$, which faces us with a dilemma where the mobile station is located. Such situation is illustrated in Fig. 5, where in comparison to Fig. 4 BS1 is moved from $(3,5)$ 
to $(6,5)$, resulting in $r_{1}=1$ for MS at $(5,5)$. Two solutions for $r_{1}$ are obtained, $r_{1 a}=1$ corresponding to MS at $(5,5)$, and $r_{1 b}=0.62887$ corresponding to MS at $(6.1134,4.3814)$. Circles that correspond to $r_{1 b}$ are shown in dashed lines. In (Bensky, 2008), a priori knowledge about the mobile station location is advised as a tool to overcame the situation. In the example of Fig. 5, it is shown that the two solutions can be close one to another, which would require significant amount of a priori knowledge to determine the location of the mobile station. The situation where two solutions are close might be expected where one of the intersecting hyperbolas is highly curved, which is caused by $|d / D|$ approaching 1 . To determine actual position of the mobile station, an additional source of information is needed, i.e. another base station that provides information about the difference in distances. However, in the case an additional source of information is available, solving of the quadratic equation is not required, and the problem could be transformed to linear (Gillette \& Silverman, 2008).

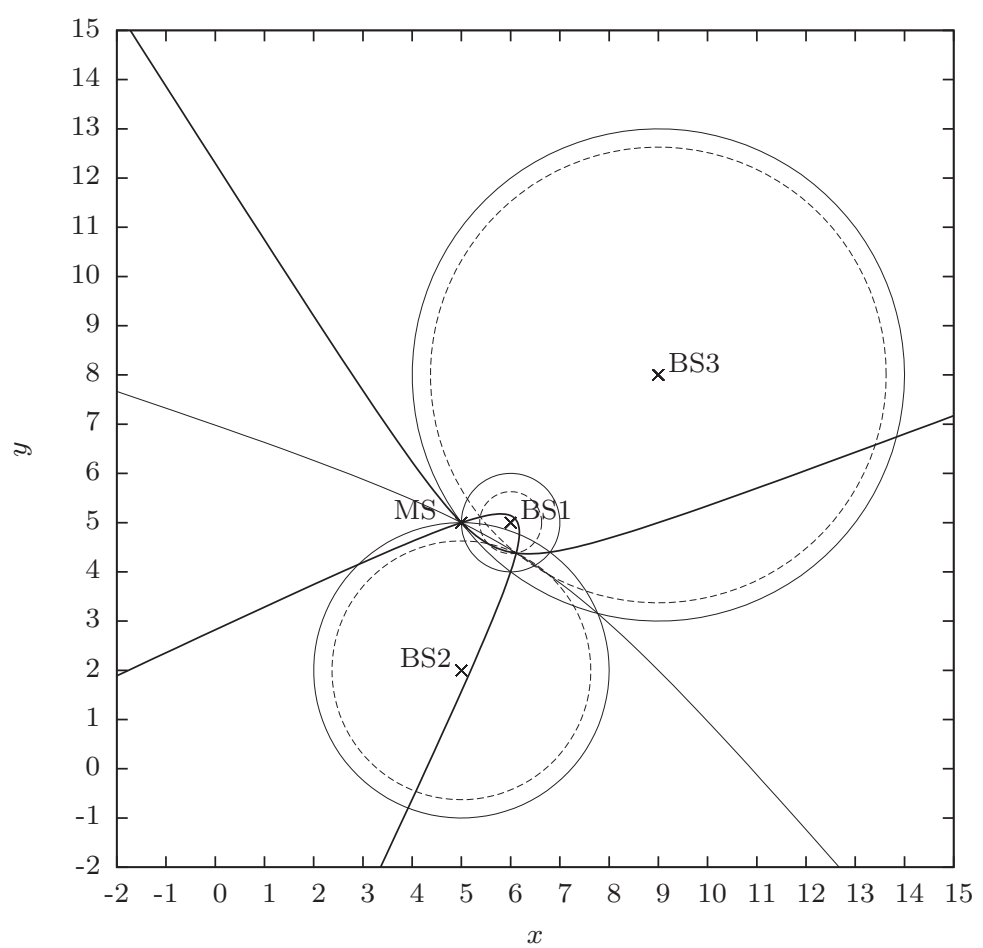

Fig. 5. Hyperbolic lateration, pair of solutions.

Let us consider (63). Variable $r_{1}$ is unknown, and it is represented by a linear term on the right hand side. Simple transfer of the term that involves $r_{1}$ to the left hand side of (63) results in

$$
2\left(x_{B S 1}-x_{B S k}\right) x_{M S}+2\left(y_{B S 1}-y_{B S k}\right) y_{M S}-2 d_{k} r_{1}=d_{k}^{2}+x_{B S 1}^{2}-x_{B S k}^{2}+y_{B S 1}^{2}-y_{B S k}^{2} .
$$


Adding the information that originates from the fourth base station, BS4, the system of linear equations over $x_{M S}, y_{M S}$, and $r_{1}$ is obtained as

$$
\left[\begin{array}{ll}
x_{B S 1}-x_{B S 2} & y_{B S 1}-y_{B S 2}-d_{2} \\
x_{B S 1}-x_{B S 3} & y_{B S 1}-y_{B S 3}-d_{3} \\
x_{B S 1}-x_{B S 4} & y_{B S 1}-y_{B S 4}-d_{4}
\end{array}\right]\left[\begin{array}{c}
x_{M S} \\
y_{M S} \\
r_{1}
\end{array}\right]=\frac{1}{2}\left[\begin{array}{c}
d_{2}^{2}+x_{B S 1}^{2}-x_{B S 2}^{2}+y_{B S 1}^{2}-y_{B S 2}^{2} \\
d_{3}^{2}+x_{B S 1}^{2}-x_{B S 3}^{2}+y_{B S 1}^{2}-y_{B S 3}^{2} \\
d_{4}^{2}+x_{B S 1}^{2}-x_{B S 4}^{2}+y_{B S 1}^{2}-y_{B S 4}^{2}
\end{array}\right] .
$$

Solution of the system provides unique information about the mobile station coordinates expressed in a closed-form. In the case more than four base stations provided information about the distance difference, an overdetermined system of equations is obtained as

$$
\left[\begin{array}{ccc}
x_{B S 1}-x_{B S 2} & y_{B S 1}-y_{B S 2}-d_{2} \\
\vdots & \vdots & \vdots \\
x_{B S 1}-x_{B S n} & y_{B S 1}-y_{B S n}-d_{n}
\end{array}\right]\left[\begin{array}{c}
x_{M S} \\
y_{M S} \\
r_{1}
\end{array}\right]=\frac{1}{2}\left[\begin{array}{c}
d_{2}^{2}+x_{B S 1}^{2}-x_{B S 2}^{2}+y_{B S 1}^{2}-y_{B S 2}^{2} \\
\vdots \\
d_{n}^{2}+x_{B S 1}^{2}-x_{B S n}^{2}+y_{B S 1}^{2}-y_{B S n}^{2}
\end{array}\right]
$$

and it can be solved in the least-squares sense (19). The method that involves the square equation over $r_{1}$ (72) should be applied in cases where information from only three base stations are available, which results in uniquely determined position of the mobile station in some cases, and in two possibilities for the mobile station location in the remaining cases.

\subsection{Probabilistic methods}

In contrast to deterministic methods that apply geometric relations to estimate the mobile station position assuming fixed distances and/or angles extracted from radio propagation parameters, probabilistic methods treat available data about the mobile station location as spatial probability density functions. This approach is suitable when accuracy of available data is poor, which frequently is the case in mobile station positioning problems in cellular networks. After the available information about the position related parameters are collected, corresponding probability density functions are joined to a single probability density function that describes position of the mobile station within the cellular network. Coordinates of the mobile station are estimated as expectation of the random variable that corresponds to the resulting probability density function. In comparison to the deterministic methods, probabilistic methods are computationally more intensive.

\subsubsection{Joining of the probability density functions}

Let us assume that an information source indexed $i$ provides information in the form of a two-dimensional probability density function $p_{i}(x, y)$ stating that probability that the mobile station is located in a rectangle specified by $x_{1}<x<x_{2}$ and $y_{1}<y<y_{2}$ is

$$
P_{i}\left(x_{1}<x<x_{2}, y_{1}<y<y_{2}\right)=\int_{y_{1}}^{y_{2}} \int_{x_{1}}^{x_{2}} p_{i}(x, y) d x d y .
$$

Probabilistic methods join the probability density functions collected from various sources of information in order to improve the information about the mobile station location. At first, let us consider two probability density functions $p_{i}(x, y)$ and $p_{j}(x, y)$. Multiplying the probability density functions provides a four-dimensional probability density function

$$
q_{i j}\left(x_{1}, y_{1}, x_{2}, y_{2}\right)=p_{i}\left(x_{1}, y_{1}\right) p_{j}\left(x_{2}, y_{2}\right)
$$


The probability density function of interest applies under constraints $x_{1}=x_{2}=x$ and $y_{1}=$ $y_{2}=y$, i.e. that both of the sources of information provide the same answer about the mobile station location. Joined probability density function is then

$$
p_{i j}(x, y)=\frac{p_{i}(x, y) p_{j}(x, y)}{\int_{-\infty}^{+\infty} \int_{-\infty}^{+\infty} p_{i}(x, y) p_{j}(x, y) d x d y} .
$$

This result generalized for $n$ probability density functions, from $p_{1}(x, y)$ to $p_{n}(x, y)$, is

$$
p(x, y)=\frac{\prod_{i=1}^{n} p_{i}(x, y)}{\int_{-\infty}^{+\infty} \int_{-\infty}^{+\infty} \prod_{i=1}^{n} p_{i}(x, y) d x d y} .
$$

Coordinates $\left(x_{M S}, y_{M S}\right)$ that most likely reveal the mobile station position are obtained as an expected value of the two-dimensional random variable $(x, y)$ with the probability density function $p(x, y)$

$$
x_{M S}=\int_{-\infty}^{+\infty} \int_{-\infty}^{+\infty} x p(x, y) d x d y=\int_{-\infty}^{+\infty}\left(\int_{-\infty}^{+\infty} p(x, y) d y\right) x d x
$$

and

$$
y_{M S}=\int_{-\infty}^{+\infty} \int_{-\infty}^{+\infty} y p(x, y) d x d y=\int_{-\infty}^{+\infty}\left(\int_{-\infty}^{+\infty} p(x, y) d x\right) y d y .
$$

As a measure of precision the mobile station coordinates are determined, standard deviation may be used,

$$
\sigma=\sqrt{\int_{-\infty}^{+\infty} \int_{-\infty}^{+\infty}\left(\left(x-x_{M S}\right)^{2}+\left(y-y_{M S}\right)^{2}\right) p(x, y) d x d y}
$$

being lower for better precision.

Coordinates of the mobile station provided computing the random variable expected value sometimes might provide an absurd result, since they may identify location of the mobile station at a point where the probability density is equal to zero, and where the mobile station cannot be located. However, the expected value provides the best guess of the mobile station location in the sense the standard deviation is minimized.

\subsubsection{Limits of the mobile station possible location}

To determine the mobile station location numerically while minimizing the computational burden, the space where the mobile station might be located should be reduced as much as possible according to the available information. Let us assume that $p_{i}(x, y)=0$ everywhere outside a rectangular region specified by

$$
x_{i \min } \leq x \leq x_{i \max }
$$

and

$$
y_{i \min } \leq y \leq y_{i \max }
$$

Inside the region, there might be points and even sub-regions where $p_{i}(x, y)=0$, but outside the rectangular region there should not be any point with $p_{i}(x, y) \neq 0$. Values of $x_{i \text { min }}$ and $y_{i \text { min }}$ should be the highest, while $x_{i \text { max }}$ and $y_{i \text { max }}$ should be the lowest values that provide $p_{i}(x, y)=0$ outside the region specified by (83) and (84). This results in the smallest rectangle 
that encloses nonzero values of the probability density function. Some probability density functions, including widely used normal distribution, take nonzero value in the entire region they are defined. In these cases, reasonable approximations should be used to neglect low nonzero values of the probability density.

According to (79), the joined probability density function takes nonzero value in points where all of the probability density functions are nonzero. Thus, the region where the joined probability density function may take nonzero value is an intersection of the rectangles specified by each of the probability density functions, given by

$$
x_{\min } \leq x \leq x_{\max }
$$

and

$$
y_{\min } \leq y \leq y_{\max }
$$

where

$$
\begin{aligned}
& x_{\text {min }}=\max _{1 \leq i \leq n}\left(x_{i \text { min }}\right) \\
& x_{\text {max }}=\min _{1 \leq i \leq n}\left(x_{i \text { max }}\right) \\
& y_{\text {min }}=\max _{1 \leq i \leq n}\left(y_{i \text { min }}\right)
\end{aligned}
$$

and

$$
y_{\max }=\min _{1 \leq i \leq n}\left(y_{i \max }\right) .
$$

In (Simić \& Pejović, 2009), a probabilistic algorithm that stops here is proposed to estimate the mobile station location, being named the method of squares. Probability density function that assumes uniform distribution within the rectangle defined by (85) and (86) is assumed, and center of the resulting rectangle

$$
x_{M S}=\frac{x_{\min }+x_{\max }}{2}
$$

and

$$
y_{M S}=\frac{y_{\min }+y_{\max }}{2}
$$

is proposed as the mobile station position estimate. This results in standard deviation of the mobile station coordinates treated as the two-dimensional random variable given by

$$
\sigma=\sqrt{\frac{\left(x_{\max }-x_{\min }\right)^{2}+\left(y_{\max }-y_{\min }\right)^{2}}{12}} .
$$

This method is computationally efficient, but suffers from poor precision, i.e. significant standard deviation (93). To improve the precision, numerical methods are applied to refine the region defined by (85) and (86).

\subsubsection{Discretization of space}

To facilitate numerical computation, the obtained region of interest specified by (85) and (86), is discretized into a grid of $n_{X}$ segments over $x$ coordinate resulting in the segment width

$$
\Delta x=\frac{x_{\max }-x_{\min }}{n_{X}}
$$


and $n_{Y}$ segments over $y$ coordinate

$$
\Delta y=\frac{y_{\max }-y_{\min }}{n_{Y}}
$$

wide. It is common to choose $n_{X}$ and $n_{Y}$ to provide $\Delta x=\Delta y$. In this manner, the space of the mobile station possible location is discretized into the grid of $n_{X} \times n_{Y}$ segments.

After the segmentation of coordinate axes is performed, let us discretize $x$ coordinate in the region of interest (85) into a vector of discrete values

$$
x_{k}=x_{\min }+\left(k-\frac{1}{2}\right) \Delta x
$$

for $k=1, \ldots n_{X}$. Discrete coordinate values of $x_{k}$ correspond to coordinates of central points of the segments. In the same manner, the discretization is performed over $y$ coordinate in the region (86),

$$
y_{l}=y_{\min }+\left(l-\frac{1}{2}\right) \Delta y
$$

for $l=1, \ldots n_{Y}$. Discretized coordinates would represent positions of grid elements in subsequent computations.

According to the space discretization and (76), the probability density functions should be integrated over each grid element to obtain the probability that the mobile station is located in that element

$$
P_{i, k, l}=\int_{y_{\min }+(l-1) \Delta y}^{y_{\min }+l \Delta y} \int_{x_{\min }+(k-1) \Delta x}^{x_{\min }+k \Delta x} p_{i}(x, y) d x d y
$$

for $i \in\{1, \ldots n\}$, which might be approximated as

$$
P_{i, k, l} \approx \Delta x \Delta y p_{i}\left(x_{k}, y_{l}\right) \text {. }
$$

In this manner, the probability density functions $p_{i}(x, y)$ are for $i \in\{1, \ldots n\}$ discretized into matrices of probabilities $P_{i, k, l}$ for $k=1, \ldots n_{X}$ and $l=1, \ldots n_{Y}$.

In terms of discretized space, elements of joined probability matrix are obtained as

$$
P_{k, l}=\frac{\prod_{i=1}^{n} P_{i, k, l}}{\sum_{k=1}^{n_{X}} \sum_{l=1}^{n_{Y}} \prod_{i=1}^{n} P_{i, k, l}} .
$$

The mobile station coordinates are obtained computing the expected value in discretized terms applying

$$
x_{M S}=\sum_{k=1}^{n_{X}}\left(\sum_{l=1}^{n_{Y}} P_{k, l}\right) x_{k}
$$

and

$$
y_{M S}=\sum_{l=1}^{n_{Y}}\left(\sum_{k=1}^{n_{X}} P_{k, l}\right) y_{l} .
$$

The standard deviation is obtained as

$$
\sigma=\sqrt{\sum_{k=1}^{n_{X}} \sum_{l=1}^{n_{Y}}\left(\left(x_{k}-x_{M S}\right)^{2}+\left(y_{l}-y_{M S}\right)^{2}\right) P_{k, l}} .
$$

By (101)-(103), computation of integrals is replaced by summations. Reducing the space of interest to the rectangular area specified by (87)-(90), the sums are made finite and with fixed limits. 


\subsubsection{Implementation of the algorithm for probability density functions of the exclusion type}

The method in its discretized form requires the probability matrix that contains $n_{X} \times n_{Y}$ entries. Handling of this matrix might be computationally inefficient in some cases, and may require significant storage space. To simplify the computation, property of some probability density functions to provide information where the mobile station cannot be located, and uniform probability density in the areas where the mobile station can be located may be utilized. These probability density functions are named probability density functions of the exclusion type (Simić \& Pejović, 2009). Typical examples are the probability density functions obtained from parameters significantly discretized in the mobile communication system, such as the timing advance (TA) parameter in GSM, and the round trip time (RTT) parameter in UMTS. After discretization, these probability density functions result in a set $S_{N Z}$ of $n_{N Z}$ grid elements, $n_{N Z} \leq n_{X} \times n_{Y}$, determined by their indices $\left(k_{j}, l_{j}\right)$ for $j=1, \ldots n_{N Z}$, where probability that the mobile station is located within the grid element takes nonzero value equal to $1 / n_{N Z}$, while it takes zero value elsewhere, i.e.

$$
P_{i, k, l}=\left\{\begin{array}{cl}
1 / n_{N Z} & \text { for }(k, l) \in S_{N Z} \\
0 & \text { for }(k, l) \notin S_{N Z}
\end{array}\right.
$$

Actual nonzero magnitude of the probability density function in the case of the probability density functions of the exclusion type is of low importance, since it can easily be computed if needed according to the normalization criterion, which after discretization for the joined probability takes form

$$
\sum_{k=1}^{n_{X}} \sum_{l=1}^{n_{Y}} P_{k, l}=1 .
$$

Thus, to store probabilities that arise from the probability density functions of the exclusion type it is enough to use one binary digit per grid element. Sources of information about the mobile station location are represented by their bitmaps $b_{i, k, l}$ that take value 1 for nonzero probability, indicating that it is possible that the mobile station is located within the corresponding grid element, and 0 for the probability equal to zero, indicating that it is not possible that the mobile station is located within the considered grid element. Besides the significant reduction in storage requirements, this simplifies joining of the probabilities obtained from different sources of information. Multiplication of probability values reduces to logical AND operation over the bits that show is it possible for the mobile station to be located within the considered grid element or not. After the joined probability bitmap is obtained as

$$
b_{k, l}=\bigwedge_{i=1}^{n} b_{i, k, l}
$$

normalization to corresponding joined probability matrix can be performed applying

$$
P_{k, l}=\frac{1}{n_{1}} b_{k, l}
$$

where $n_{1}$ is the number of elements in $b_{k, l}$ that take value 1 ,

$$
n_{1}=\sum_{k=1}^{n_{X}} \sum_{l=1}^{n_{Y}} b_{k, l} .
$$


The normalization should not be performed at the matrix level, since it is more convenient to perform it on the level of coordinate and standard deviation computation,

$$
\begin{aligned}
& x_{M S}=\frac{1}{n_{1}} \sum_{k=1}^{n_{X}}\left(\sum_{l=1}^{n_{Y}} b_{k, l}\right) x_{k} \\
& y_{M S}=\frac{1}{n_{1}} \sum_{l=1}^{n_{Y}}\left(\sum_{k=1}^{n_{X}} b_{k, l}\right) y_{l}
\end{aligned}
$$

and

$$
\sigma=\sqrt{\frac{1}{n_{1}} \sum_{k=1}^{n_{X}} \sum_{l=1}^{n_{Y}}\left(\left(x_{k}-x_{M S}\right)^{2}+\left(y_{l}-y_{M S}\right)^{2}\right) b_{k, l}} .
$$

Sometimes it might be useful to approximate the probability density functions that are not of the exclusion type by the exclusion type ones, sacrificing some of the precision in order to improve the computational efficiency. Besides, the bitmap of (106) provides useful visual information that can be presented to the user as a map of the mobile station possible location.

\subsubsection{An example}

To illustrate application of probabilistic methods, an example that includes two base stations of a GSM network located at $\left(x_{B S 1}, y_{B S 1}\right)=(0,0)$ with $T A=1$ and $\left(x_{B S 2}, y_{B S 2}\right)=$ $\left(1.8 R_{q}, 1.6 R_{q}\right)$ with $T A=0$ is created, as shown in Fig. 6 . All of the distances considered in this example are expressed in terms of the GSM spatial resolution quantum $R_{q}=553.46 \mathrm{~m} \approx$ $550 \mathrm{~m}$. Line-of-sight wave propagation is assumed, thus probabilistic model of (7) is applied. Under these assumptions, according to (10), (11), and (87)-(90), the space where the mobile station might be located is limited to $0.8 R_{q} \leq x_{M S} \leq 2 R_{q}$ and $0.6 R_{q} \leq y_{M S} \leq 2 R_{q}$. To perform discretization of space, the same discretization quantum is applied for both of the axes, $\Delta x=\Delta y=0.1 R_{q}$. In Fig. 6, in the region of the mobile station possible location spatial grid of $n_{X} \times n_{Y}=12 \times 14=168$ elements is drawn, and center points of the grid elements are indicated by dots. The probability density function of (7) is of the exclusion type, and applying the algorithm for this class of functions the grid elements are classified regarding possible position of the mobile station. To perform this task, distance between two points had to be determined $2 \times 168=336$ times. Grid elements where the mobile station might be located are shaded in Fig. 6. Applying (109) and (110), the mobile station coordinates are estimated as $x_{M S}=1.2935 R_{q}$ and $y_{M S}=1.1471 R_{q}$, while according to (111) standard deviation of the location estimation is obtained as $\sigma=0.40556 R_{q}$. For comparison, the method of squares (91)-(93) provides $x_{M S}=1.4 R_{q}$ and $y_{M S}=1.3 R_{q}$ with $\sigma=0.53229 R_{q}$, without any need to compute distances mentioned 336 times.

To apply deterministic methods in order to provide a comparison, coordinates of two base stations and distances to the mobile station estimated according to (12) as $r_{1}=1.5 R_{q}$ and $r_{2}=$ $0.5 R_{q}$ are available, as well as the distance difference $d_{2}=r_{2}-r_{1}=R_{q}$. The available set of data is not sufficient to determine position of the mobile station. However, some information about the mobile station position might be extracted.

For circular lateration, the distance between the base stations $D=2.40832 R_{q}$ is larger than $r_{1}+r_{2}=2 R_{q}$, thus the circles of possible mobile station location do not intersect. However, equation of the type (24) locates the mobile station on a line $18 x+16 y=39$, which is shown 


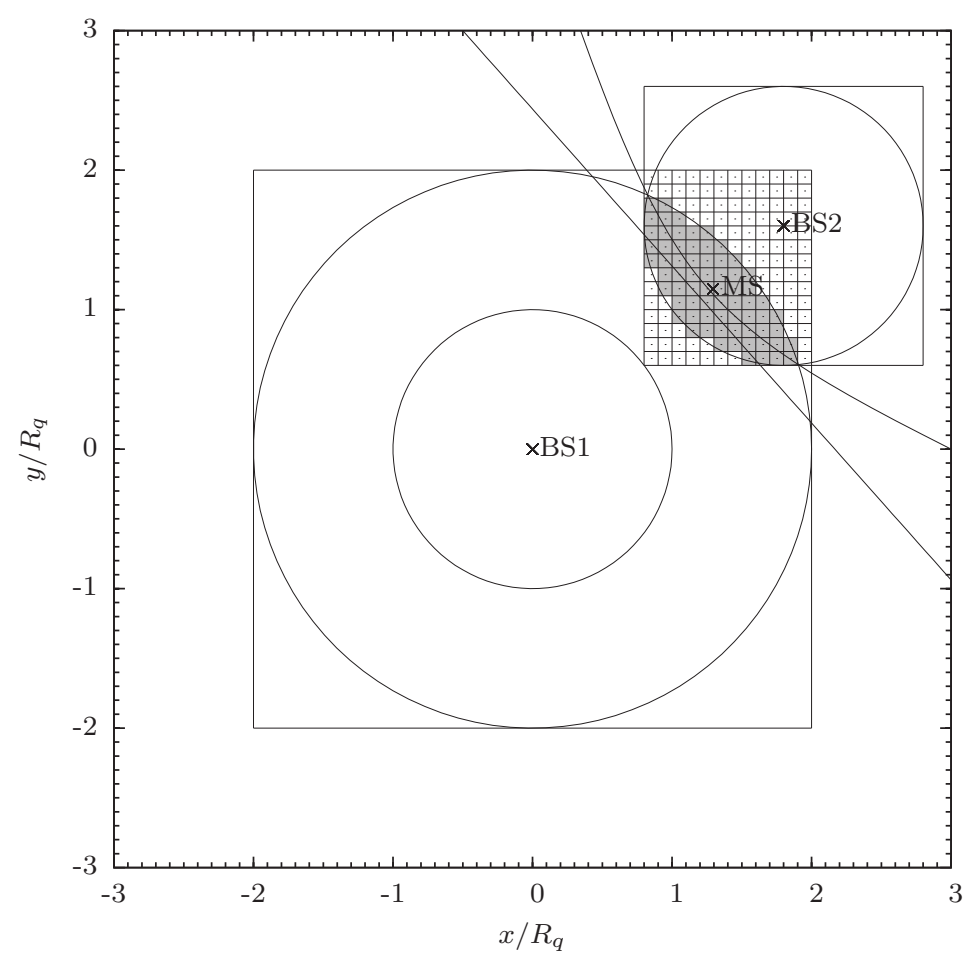

Fig. 6. Probabilistic methods, an example.

in Fig. 6. On the other hand, hyperbolic lateration locates the mobile station on a hyperbola obtained applying (53) as

$$
x=0.9+0.3737 \cosh t-0.7278 \sinh t
$$

and

$$
y=0.8+0.3322 \cosh t+0.8187 \sinh t .
$$

The hyperbola passes through the intersections of the outer boundary circles of the regions defined by (7), which have the radii for $\frac{1}{2} R_{q}$ higher than the estimated distances of the mobile station from corresponding base stations. In the distance difference, these offsets cancel out, thus the hyperbola passes through the intersections of circles, as it would pass through the intersections of circles that represent estimated distance to the mobile station if they had intersected.

As illustrated in this example, probabilistic approach is able to provide an estimate of the mobile station coordinates even in cases when available data is insufficient for deterministic methods. However, probabilistic methods are computationally more intensive.

\subsection{Fingerprinting methods}

Fingerprinting methods (Küpper, 2005) treat a vector of position related parameters that mobile station observes as a fingerprint of the mobile station position. The position is 
determined comparing the observed vector to vectors stored in a predetermined database of fingerprints. The database is obtained either by field measurements or precomputed applying appropriate wave propagation models. Point in the database having the vector of position related parameters the closest to the vector observed by the mobile station is assumed as the mobile station position.

Radio propagation parameter convenient to be used in a vector of position related parameters is the received signal power. This is particularly convenient for application in WLAN networks (Küpper, 2005). Let us assume that applying some information about the mobile station location (the user is in a building, ID of the serving cell is known, etc.) the set of base stations that might be observed by the mobile station is reduced to a set of $n$ base stations. Observed vector of position related parameters is

$$
\vec{P}=\left[P_{O 1}, \ldots P_{O n}\right] .
$$

Let us also assume that a database of $M$ entries is predetermined, consisting of vectors

$$
\vec{P}_{m}=\left[P_{D B m, 1}, \ldots P_{D B m, n}\right]
$$

accompanied by corresponding coordinates $\left(x_{m}, y_{m}\right)$ for $m \in\{1, \ldots M\}$. As a measure of difference between the observed vector of position related parameters, Euclidean distance

$$
\delta_{m}^{2}=\left|\vec{P}-\vec{P}_{m}\right|^{2}=\sum_{k=1}^{n}\left(P_{O k}-P_{D B m, k}\right)^{2}
$$

might be used. Index $m_{M S}$ of the database entry that corresponds to the mobile station position is obtained as

$$
\delta_{m_{M S}}^{2}=\min _{1 \leq m \leq M} \delta_{m}^{2}
$$

and the mobile station coordinates are obtained as $\left(x_{m_{M S}}, y_{m_{M S}}\right)$. The problem that might appear here is that (117) might provide multiple solutions for $m_{M S}$, likelihood of which is increased by rougher quantization of the received signal power. The situation would not be frequent in well designed systems, with adequate choice of the vector of position related parameters. In the case the vector of position related parameters contains variables with different physical dimensions, to enable determination of Euclidean distance normalization, i.e. nondimensionalization of parameters, should be performed. This introduces weighting coefficients for the elements of the vector of position related parameters, which can be introduced even in the case the elements of the vector are of the same physical dimension.

Described fingerprinting method for each positioning request requires Euclidean distance computation and minimum search over the entire database. This might be computationally intensive and time consuming. To simplify the database search, in (Simić \& Pejović, 2008) a set of $n_{C B S}$ base stations with the strongest received signal power is proposed as a fingerprint for GSM networks. The position fingerprint contains only indexes of the base stations, not the information about the received signal power. Since the information about received signal power of up to seven base stations observed by the mobile station is included in standard measurement report in GSM, it is convenient that $1 \leq n_{C B S} \leq 7$. According to the set of base stations with the highest received signal power, the space is divided into segments characterized by the same fingerprint. Within the segment, the probabilistic approach is applied assuming uniform probability density function. The information each fingerprint provides are estimated coordinates of the mobile station, precomputed as mathematical 
expectation of the coordinates treated as a random variable of uniform probability density within the segment, corresponding standard deviation, and the map of the segment. In (Simić \& Pejović, 2008), a simple propagation model that assumes line-of-sight propagation and monotonous decrease of signal power with distance is applied to illustrate the method. However, more complex propagation models might be applied, as well as field measurements. Search for the fingerprint in the database might be performed as a binary search, and it is computationally efficient. The method could be generalized to other cellular networks. Furthermore, the method could be extended, and instead of the set of base stations with the strongest received signal power, the fingerprint might consist of indexes of the base stations ordered according to the received signal power. Again, information about the actual received signal power is not included in the fingerprint. This approach would increase the number of available fingerprints and reduce the segment size.

\section{Standardized positioning methods in cellular networks}

Implementation of the location based services required special standards to be developed. The standardization is performed by The Third Generation Partnership Project (3GPP).

For GSM cellular networks, the following positioning methods have been standardized (3GPP TS 43.059, 2007):

- Cell-ID+TA (Cell Identification + Timing Advance),

- E-OTD (Enhanced Observed Time Difference),

- U-TDOA (Uplink Time Difference of Arrival), and

- A-GNSS (Assisted Global Navigation Satellite System).

For UMTS cellular networks, the following positioning methods have been standardized (3GPP TS 25.305, 2007):

- Cell-ID (Cell Identification),

- OTDOA-IPDL (Observed Time Difference Of Arrival-Idle Period Downlink),

- A-GNSS (Assisted Global Navigation Satellite System), and

- U-TDOA (Uplink Time Difference of Arrival).

Cell-ID is the easiest and most widespread method to obtain the location of the mobile station in cellular networks. This method is based on proximity sensing principle, i.e. estimated coordinates of the mobile station are geographic coordinates of the base station (Node B) currently serving the mobile station. A drawback of this method is poor accuracy, dependent on the serving cell size.

To achieve location estimation in GSM networks with higher accuracy, Cell-ID is combined with the timing advance value resulting in Cell-ID+TA positioning method. The TA parameter value is related to the wave propagation round trip time, which is proportional to the distance from the serving base station to the mobile station, and thus the location of the mobile station can be constrained to a ring centered at the base station.

E-OTD method is based on measurements of the signal propagation time on the downlink, and applies hyperbolic lateration. In E-OTD, the mobile station measures the time difference of arrival (TDOA) of the signals from the neighboring base stations, which are not synchronized in GSM. Hence, it is necessary to install additional components in the network to deal with the synchronization issues. These components are called Location Measurement Units (LMU). 
OTDOA is an equivalent to E-OTD positioning method in GSM. The differences are caused by different structure of UMTS radio interface. Timing measurements at the mobile station in the systems based on CDMA principle suffer from so-called hearability problem. This problem occurs when the mobile station is located near the service base station, which may block signals from other base stations that operate on the same frequency. In order to overcome this problem, each base station must interrupt its transmission in a short period of time to allow the mobile station to detect signals from neighboring base stations and to perform all the measurements necessary for lateration. These periods are called idle periods, while the mechanism that controls them is called IPDL (Idle Period Downlink). OTDOA positioning method which applies IPDL is called OTDOA-IPDL positioning method.

U-TDOA is a method standardized for positioning both in GSM and UMTS. Similarly to E-OTD and OTDOA, U-TDOA positioning method is based on measurements of the signal propagation time and applies hyperbolic lateration. However, in U-TDOA the measurements are made on the uplink, i.e. the time of arrival of the signal emitted from the mobile station is observed by the serving base station and a number of LMUs. Thus, this method also requires installation of additional hardware components, LMUs, and in larger number than in the case of E-OTD or OTDOA method.

A-GNSS is a method standardized for positioning both in GSM and UMTS cellular networks. Unlike the positioning methods previously described, completely based on the cellular network infrastructure, A-GNSS positioning method is based on the satellite infrastructure of the Global Positioning System, GPS. This method requires mobile stations to be equipped with a GPS receiver, and they are supplied by some additional assistance data from the network, which allow to reduce the acquisition time and to increase the accuracy.

\section{Conclusions}

Methods that utilize propagation parameters of radio waves to determine mobile station location are analyzed in this chapter. Propagation parameters of radio waves are briefly analyzed, and it is shown that distance can be estimated from the propagation path loss and from the wave propagation time. To measure the wave propagation time, clocks involved in the measurement should be synchronized. In the case the distance between two points is measured, to avoid synchronization the round trip time might be measured, which involves only one clock. In the case the base stations are synchronized, the time difference of the signal propagation between the mobile station and two base stations can be measured without the requirement for mobile station to be synchronized. Besides distances and differences in distance, angles of signal arrival might be used to perform positioning.

After the wave propagation data are collected, they should be processed to provide estimate of the mobile station location. Estimation algorithms are based either on deterministic or probabilistic principles. Deterministic algorithms that process the angle of signal arrival, the path loss or the wave propagation time, and the time difference of signal propagation are analyzed, named after their geometrical interpretations as angulation, circular lateration, and hyperbolic lateration, respectively. It is shown that in all three of the cases coordinates of the mobile station could be computed as a solution of a system of linear equations. To provide unique solution for the mobile station coordinates in two dimensions, it is shown that angulation requires data from at least two base stations, circular lateration from three, and hyperbolic lateration from four base stations. Additional data might be incorporated to refine the estimate of the mobile station coordinates applying linear least-squares method to solve the resulting overdetermined system of linear equations. 
Probabilistic methods model information about the mobile station location extracted from the wave propagation parameters as probability density functions. A method to process the probability density functions in order to increase the positioning precision is presented. An emphasis to computational efficiency is made. In order to reduce the memory requirement and to simplify the computation, probability density functions of the exclusion type were introduced. Application of the proposed probabilistic method results in estimated coordinates, corresponding standard deviation, and a map of the mobile station possible location. Probabilistic methods are convenient in cases where available information about the mobile station location is poor.

The third group of the methods to process the position related data analyzed in this chapter are fingerprinting methods. These methods reduce the problem to database search, treating collected set of radio propagation position related parameters as a position fingerprint. The database might be formed either applying deterministic or probabilistic methods, or even applying field measurements. Variants of the method that use the fingerprint formed as a vector of received signal power from various base stations and the fingerprint formed as a set of base station indexes formed according to the received signal power are analyzed.

A brief review of standardized positioning methods in GSM and UMTS systems is given.

\section{References}

3GPP TS 43.059, release 8 (v8.0.0), /EDGE Radio Access Network, Functional stage 2 description of Location Services (LCS) in GERAN, 2007.

3GPP TS 25.305, release 8 (v8.0.0), Stage 2 Functional specification of UE positioning in UTRAN, 2007.

Bensky, A. (2008). Wireless Positioning Technologies and Applications, Artech House, ISBN 978-1-59693-130-5, Norwood.

Bronshtein, I. N.; Semendyayev, K. A.; Musiol, G. \& Muehlig, H. (2007). Handbook of Mathematics, Springer, ISBN 978-3-540-72121-5, Berlin, Heidelberg, New York.

Gillette, M. D. \& Silverman, H. F. (2008). A linear closed-form algorithm for source localization from time-differences of arrival. IEEE Signal Processing Letters, Vol. 15, No. 1, Jan. 2008, 1-4, ISSN 1070-9908

Küpper, A. (2005). Location-based Services, John Wiley \& Sons, ISBN 978-0-470-09231-6, Chichester.

Press, W. H.; Teukolsky S. A.; Vetterling, W. T. \& Flannery, B. P. (1992). Numerical Recipes in C, Cambridge University Press, ISBN 0-521-43108-5, Cambridge.

Rappaport, T. S. (2001). Wireless communications principles and practice, Prentice Hall, ISBN 0130422320, Englewood Cliffs.

Simić, M. \& Pejović, P. (2008). An algorithm for determining mobile station location based on space segmentation. IEEE Communications Letters, Vol. 12, No. 7, July 2008, 499-501, ISSN 1089-7798

Simić, M. \& Pejović, P. (2009). A probabilistic approach to determine mobile station location with application in cellular networks. Annals of Telecommunications, Vol. 64, No. 9-10, Oct. 2009, 639-649, ISSN 0003-4347 (Print), 1958-9395 (Online)

Simić, M. \& Pejović, P. (2009). A comparison of three methods to determine mobile station location in cellular communication systems. European Transactions on Telecommunications, Vol. 20, No. 8, Dec. 2009, 711-721, ISSN 1124-318X (Print), 1541-8251 (Online) 


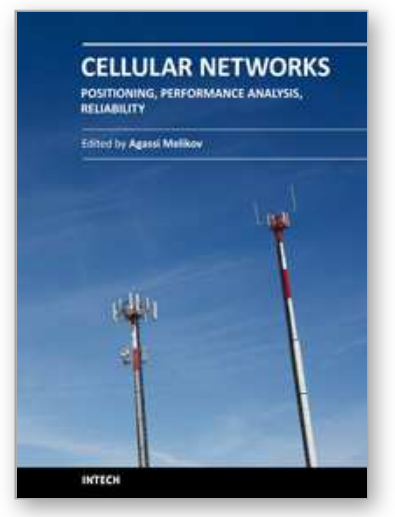

\author{
Cellular Networks - Positioning, Performance Analysis, Reliability \\ Edited by Dr. Agassi Melikov
}

ISBN 978-953-307-246-3

Hard cover, 404 pages

Publisher InTech

Published online 26, April, 2011

Published in print edition April, 2011

Wireless cellular networks are an integral part of modern telecommunication systems. Today it is hard to imagine our life without the use of such networks. Nevertheless, the development, implementation and operation of these networks require engineers and scientists to address a number of interrelated problems. Among them are the problem of choosing the proper geometric shape and dimensions of cells based on geographical location, finding the optimal location of cell base station, selection the scheme dividing the total net bandwidth between its cells, organization of the handover of a call between cells, information security and network reliability, and many others. The book focuses on three types of problems from the above list Positioning, Performance Analysis and Reliability. It contains three sections. The Section 1 is devoted to problems of Positioning and contains five chapters. The Section 2 contains eight Chapters which are devoted to quality of service (QoS) metrics analysis of wireless cellular networks. The Section 3 contains two Chapters and deal with reliability issues of wireless cellular networks. The book will be useful to researches in academia and industry and also to post-gradute students in telecommunication specialitiies.

\title{
How to reference
}

In order to correctly reference this scholarly work, feel free to copy and paste the following:

Mirjana Simić and Predrag Pejović (2011). Positioning in Cellular Networks, Cellular Networks - Positioning, Performance Analysis, Reliability, Dr. Agassi Melikov (Ed.), ISBN: 978-953-307-246-3, InTech, Available from: http://www.intechopen.com/books/cellular-networks-positioning-performance-analysis-reliability/positioning-incellular-networks

\section{INTECH}

open science | open minds

\section{InTech Europe}

University Campus STeP Ri

Slavka Krautzeka 83/A

51000 Rijeka, Croatia

Phone: +385 (51) 770447

Fax: +385 (51) 686166

www.intechopen.com

\section{InTech China}

Unit 405, Office Block, Hotel Equatorial Shanghai

No.65, Yan An Road (West), Shanghai, 200040, China 中国上海市延安西路65号上海国际贵都大饭店办公楼 405 单元

Phone: +86-21-62489820

Fax: $+86-21-62489821$ 
(C) 2011 The Author(s). Licensee IntechOpen. This chapter is distributed under the terms of the Creative Commons Attribution-NonCommercialShareAlike-3.0 License, which permits use, distribution and reproduction for non-commercial purposes, provided the original is properly cited and derivative works building on this content are distributed under the same license. 